\title{
The expert patient: towards a novel definition
}

\author{
Jean-François Cordier
}

Affiliations: Dept of Respiratory Diseases, National Reference Center for Rare Pulmonary Diseases, Louis Pradel University Hospital, Claude Bernard University, Lyon, France.

Correspondence: Jean-François Cordier, Respiratory Medicine, Louis Pradel Hospital, 28 avenue Doyen Lepine, Lyon (Bron) 69677, France. E-mail: jean-francois.cordierdachu-lyon.fr

○ @ERSpublications

Experience of illness and knowledge of disease make the expert patient an empowered partner for health professionals http://ow.ly/x8vbj

Chronic diseases have become a major burden in many developed countries. Physicians and policy makers manage patients and set clinical as well as research goals. In recent years, however, it has become progressively apparent that, as the patients are the ones living with the disease, their views and wishes should be considered, and healthcare should be more patient-centred. Many guidelines stress the importance of patient education, especially now that patients have easy access to information through the internet, mass media, educational activities in hospitals, communities and patient groups. Those living with common conditions such as chronic obstructive pulmonary disease (COPD) and asthma, and even rare pulmonary disorders, progressively acquire both experience and knowledge of their condition. The importance of patient involvement not only in their own management but also in health policy planning and decision-making is now being emphasised in Europe. Task forces are beginning to include patients in their expert panels when setting guidelines.

\section{Patient education and self-management of chronic illnesses/diseases Illness and disease are not synonymous}

Medical anthropologists use the terms "illness" and "disease" to describe the different views of health held by patients and doctors, respectively [1]. "Illness" not only includes the experiences of ill health but also the meaning given to these experiences, which are influenced by the patient's social and cultural background, including "folk" models of illness [1,2]. "Disease" is an abnormality in the function and/or structure of the body's organs and systems. Diseases can occur in the absence of illnesses (e.g. asymptomatic arterial hypertension).

In brief, patients suffer illnesses, and physicians diagnose and treat their diseases $[1,3,4]$.

\section{Patient education and self-management}

Patient education consists of healthcare professionals teaching patients about their disease and offering therapeutic instruction, particularly information about drugs, adaptation of their dosage and side-effects. Patient education is the first step on the way to self-management.

Patient self-management [5] is the individual's ability to control their symptoms, treatment, the physical and psychological consequences of their condition, and the lifestyle or behaviour associated with chronic conditions [6]. The objective of self-management is to control the condition with clear strategies to achieve desired outcomes [5]. Interventions may be delivered by a variety of health professionals, including physicians, nurses, psychologists, educators, etc. In asthma, most interventions target some aspect of behaviour, particularly compliance with prophylactic treatment [5]. 


\section{Chronic disease self-management programmes}

Since the 1980s, Kate Lorig of Stanford University (Stanford, CA, USA) has been developing chronic disease self-management programmes (CDSMP) for conditions such as arthritis, lung disease, heart disease and stroke. All of her interventional work is based on the theory of self-efficacy [7] expounded by Albert Bandura [8]. Self-efficacy is confidence in one's ability to carry out an action in order to achieve a defined goal. Self-management does not consist of solving a particular problem but in learning how to solve it. Selfefficacy is therefore important in behaviour change, as it reinforces perseverance in self-management thereby improving health and well-being.

The originality of most studies by Kate Lorig and her colleagues resides in the leadership of trained, volunteer and lay people who themselves have at least one chronic disease requiring intervention $[9,10]$.

A review of the Stanford CDSMP disclosed minor, statistically significant improvements in health status outcomes, healthy behaviour and self-efficacy [11]. Another review of 17 trials involving 7442 participants concluded that lay-led self-management education programmes elicit slight, short-term improvements in participants' self-efficacy, self-rated health, cognitive symptom management and aerobic exercise frequency [12]. Therefore, improvement due to CDSMP mainly impacts on illness.

Interestingly, CDSMP that are accessible via the internet are effective as they can reach those in rural areas as well as those who have insufficient access to their healthcare system [13-15]. However, internet-based interventions are limited to those who are literate and have internet access [15].

\section{Expert patient programmes}

The term "expert patient" first appeared in a report presented to the UK Parliament in 1999 as a "healthy citizen" initiative to help deal with chronic illness [16]. This initiative was further developed in report by the UK's Department of Health [17]. The report suggested that expert patient programmes in the UK should be based on: "developing the confidence and motivation of patients to use their own skills and knowledge to take effective control over life with a chronic illness" [17]. Recommendations included the promotion of awareness, the creation of expectations that patient expertise is a central component in the delivery of care, the development of more user-led CDSMP, and the creation of a care course that advances healthcare professionals' knowledge of user-led self-management programmes.

UK expert patient programmes, which were based on CDSMP and adapted to the internet, appeared to decrease symptoms and improve health behaviours, self-efficacy and satisfaction with the healthcare system, reducing healthcare utilisation by up to 1 year [13].

\section{The novel expert patient: illness experience and disease expertise}

Experts are people who are very knowledgeable about or skilful in particular areas. Knowledge of relevant facts, experience in a given field, perceptiveness and communication skills are some of the features of expertise [18].

Expert patients should be able to both help other patients will illness self-management and work with physicians in ensuring the development of comprehensive care and disease management (table 1). Expert patients cannot play a solitary role but must develop an expert-team approach to chronic illness/disease.

Expert patients have the unique opportunity to clarify patient values and priorities, which in turn may better inform clinical decision-making.

\section{From individual to collective experience}

As holders of personal and experiential knowledge about their illness, expert patients are adept in identifying some needs that are not considered or are only poorly considered by doctors and other healthcare professionals. Their own experience may be further enriched by that of other patients (especially in the context of patients' associations), resulting in collective and, thus, greater experience of illness.

\section{Expertise in common and rare diseases}

Knowledge about common diseases, such as asthma, amongst general practitioners and respiratory specialists is very good. Medical knowledge about this condition has long been acquired by patients and the public, and is disseminated by the lay press and other mass media.

In contrast, patients with rare conditions have often been orphans in the world of disease. Family physicians have limited knowledge of the more than 5000 rare, existent diseases, and even specialists encounter only a few of these patients. The designation of national expert centres for rare diseases in European countries has played a major part in research development and specialised care, as have regional centres and the participation of patients' associations, resulting in efficient networking. 
TABLE 1 Experiential and academic skills that define expert patients

Experiential

Personal knowledge of illness and treatment

Education as a patient, including self-management

Collaborative relationship with the general practitioner and proximity specialist

Membership of patients' associations

Attendance at local patient meetings

Participation (as a patient) in clinical studies/ therapeutic trials

\section{Academic}

Knowledge of the disease and treatment

Academic education as an educator/teacher

Participation as an educator/teacher with health professionals in patient education, including selfmanagement, and taking into account patient values and priorities for clinical decision-making

Collaborative relationship with academic specialists

Responsibilities in patients' associations (e.g. as a board member)

Attendance and active participation in regional/ national/international patient meetings

Participation as a partner in the design of clinical studies/therapeutic trials

This list is not exhaustive (e.g. expert patient participation in physician education should be considered).

I believe that the definition of an expert patient should include both experiential (personal and collective) knowledge of illness as well as pertinent awareness of diseases and academic involvement. Such patients could increasingly fulfil diverse functions not only in patient education and self-management, but also in the education of healthcare professionals, especially doctors and nurses, who sometimes neglect the importance of illness and the experiential knowledge of patients by focusing only on disease.

\section{Extending the possible role of expert patients}

Are patients guinea pigs or partners in trials?

Patients are "participants" or "study subjects" in therapeutic trials. Their informed consent is solicited but is often a mere formality. Expert patients should be invited to comment on the objectives of planned trials, participate in study design of and in discussion about the results (especially side-effects and the risk:benefit ratio). The side-effects of new drugs are often poorly detailed in print, and only rarely is their management analysed.

In contrast to the traditional paternalistic approach to patient care, empowerment allows mastery by people over their affairs; empowering patients therefore promotes autonomous self-regulation. Empowered people have sufficient knowledge, control and resources to make rational decisions, implement them and evaluate their effectiveness $[19,20]$. Empowerment of expert patients as true partners in clinical studies and therapeutic trials has become an ethical necessity.

\section{Engagement in safety}

As patients are the only individuals present during every consultation and treatment, they could be uniquely positioned as vigilant monitors of safety [21], especially at the bedside during the final stage of treatment. They can detect medication administration errors, including drugs intended for other patients [22], lack of hand hygiene [23], malfunction of devices (especially perfusion pumps), etc. Although patients' attitudes towards engaging in error prevention vary considerably, when clinicians themselves encourage patients' involvement in safety, most of them become willing participants [24]. This attitude could be further enhanced in expert patients.

\section{Patients' neglected concerns}

Healthcare professionals are not always aware of patients' concerns. Stress incontinence, which occurs mainly in females, is very common during coughing spells in cystic fibrosis [25], COPD [26] and interstitial lung disease patients [27]. Patients seldom speak spontaneously to their physicians because of feelings of shame [27]. Stress incontinence is typically an embarrassing manifestation neglected by doctors, and expert patients could engage both doctors and patients for early and systematic recognition and treatment. Listing patients' neglected concerns is necessary. 


\section{Expert patient proxy}

Information about the exponential advances in medical and scientific knowledge in recent years is readily available on the internet, often resulting in the fragmentation of information and possibly increasing complexity. This is true for both doctors and patients, and includes ethical concerns generated by scientific progress (especially in the field of genetics). Patients with a lower education level, who are children, or who are older with cognitive limitations imposed by acquired (or inherited) diseases will have difficulty deciding in favour of or against a given intervention. In such situations, expert patients may be valid advisers by proxy, helping patients (and/or their families) to decide whether to opt for an intervention.

\section{Participatory medicine}

Participatory medicine is the active engagement of networked patients in decisions about treatment courses, reflecting data on their experiences and outcomes back to the healthcare system. Participatory studies (for example, the study by WeITZMAN et al. [28], which elucidates the burden of hypoglycaemia within an online social diabetes network) are an area in which expert patients might play a specific role in the design and follow-up of investigations or trials, in close collaboration with physicians.

\section{Conclusion}

Appropriate empowerment of patients is increasingly challenging the paternalistic dominance of physicians over patients. Self-management programmes, and in particular therapeutic education and information available on the internet, have markedly improved the knowledge that patients have of their illness and of the diseases afflicting them. The role of expert patients is particularly crucial and specific in rare diseases. Empathic and skilful expert patients are evidently necessary to the future of the healthcare system.

\section{Acknowledgements}

The author thanks V. Cottin (Louis Pradel Hospital, Lyon, France), C. Durand (Association FLAM (France Lymphangioléiomyomatose), Saint Benoit, France) and K. Mandl (Boston Children's Hospital, Harvard Medical School, Boston, MA, USA) for their pertinent comments.

\section{References}

Helman CG. Disease versus illness in general practice. J R Coll Gen Pract 1981; 31: 548-552.

Saunders L, Hewes GW. Folk medicine and medical practice. J Med Educ 1953; 28: 43-46.

Eisenberg L. Disease and illness. Distinctions between professional and popular ideas of sickness. Cult Med Psychiatry 1977; 1: 9-23.

Parry KK. Concepts from medical anthropology for clinicians. Phys Ther 1984; 64: 929-933.

Newman S, Steed L, Mulligan K. Self-management interventions for chronic illness. Lancet 2004; 364: 1523-1537. Barlow J, Wright C, Sheasby J, et al. Self-management approaches for people with chronic conditions: a review. Patient Educ Couns 2002; 48: 177-187.

7 Gilkey M, Garcia C. What do you think of when you think of patient education? An interview with Kate Lorig. Health Promot Pract 2010; 11: 169-172.

8 Bandura A. Self-efficacy: toward a unifying theory of behavioral change. Psychol Rev 1977; 84: 191-215.

9 Lorig KR, Sobel DS, Stewart AL, et al. Evidence suggesting that a chronic disease self-management program can improve health status while reducing hospitalization: a randomized trial. Med Care 1999; 37: 5-14.

10 Ory MG, Ahn S, Jiang L, et al. National study of chronic disease self-management: six-month outcome findings. J Aging Health 2013; 25: 1258-1274.

11 Franek J. Self-management support interventions for persons with chronic disease: an evidence-based analysis. Ont Health Technol Assess Ser 2013; 13: 1-60.

12 Foster G, Taylor SJ, Eldridge SE, et al. Self-management education programmes by lay leaders for people with chronic conditions. Cochrane Database Syst Rev 2007; 4: CD005108.

13 Lorig KR, Ritter PL, Dost A, et al. The Expert Patients Programme online, a 1-year study of an Internet-based selfmanagement programme for people with long-term conditions. Chronic Illn 2008; 4: 247-256.

14 Lorig K, Laurent DD, Plant K, et al. The components of action planning and their associations with behavior and health outcomes. Chronic Illn 2014; 10: 50-59.

15 Lorig K, Ritter PL, Plant K, et al. The South Australia health chronic disease self-management Internet trial. Health Educ Behav 2013; 40: 67-77.

16 Department of Health. Saving lives: our healthier nation. London, The Stationery Office, 1999.

17 Department of Health. The expert patient: a new approach to chronic disease management in the 21st century. London, Department of Health, 2001.

18 Shanteau J, Weiss DJ, Thomas RP, et al. Performance-based assessment of expertise: how to decide if someone is an expert or not. Eur J Oper Res 2002; 136: 253-263.

19 Funnell MM, Anderson RM, Arnold MS, et al. Empowerment: an idea whose time has come in diabetes education. Diabetes Educ 1991; 17: 37-41.

20 Arnold MS, Butler PM, Anderson RM, et al. Guidelines for facilitating a patient empowerment program. Diabetes Educ 1995; 21: 308-312.

21 Schwappach DL. Review: engaging patients as vigilant partners in safety: a systematic review. Med Care Res Rev 2010; 67: 119-148.

22 Kullberg A, Larsen J, Sharp L. "Why is there another person's name on my infusion bag?" Patient safety in chemotherapy care - a review of the literature. Eur J Oncol Nurs 2013; 17; 228-235. 
McGuckin M, Govednik J. Patient empowerment and hand hygiene, 1997-2012. J Hosp Infect 2013; 84: 191-199. Doherty C, Stavropoulou C. Patients' willingness and ability to participate actively in the reduction of clinical errors: a systematic literature review. Soc Sci Med 2012; 75: 257-263.

Parkins MD, Parkins VM, Rendall JC, et al. Changing epidemiology and clinical issues arising in an ageing cystic fibrosis population. Ther Adv Respir Dis 2011; 5: 105-119.

Hrisanfow E, Hagglund D. The prevalence of urinary incontinence among women and men with chronic obstructive pulmonary disease in Sweden. J Clin Nurs 2011; 20: 1895-1905.

Bradaia F, Lazor R, Khouatra C, et al. Urinary incontinence due to chronic cough in interstitial lung disease. Rev Mal Respir 2009; 26: 499-504.

Weitzman ER, Kelemen S, Quinn M, et al. Participatory surveillance of hypoglycemia and harms in an online social network. JAMA Intern Med 2013; 173: 345-351. 